\title{
Urogenital pathogens, associated with Trichomonas vaginalis, among pregnant women in Kilifi, Kenya: a nested case- control study
}

\author{
Simon C. Masha ${ }^{1,2,3^{*}}$ (D), Piet Cools ${ }^{2}$, Patrick Descheemaeker ${ }^{4}$, Marijke Reynders ${ }^{4}$, Eduard J. Sanders ${ }^{1}$
} and Mario Vaneechoutte ${ }^{2}$

\begin{abstract}
Background: Screening of curable sexually transmitted infections is frequently oriented towards the diagnosis of chlamydia, gonorrhea, syphilis and trichomoniasis, whereas other pathogens, sometimes associated with similar urogenital syndromes, remain undiagnosed and/or untreated. Some of these pathogens are associated with adverse pregnancy outcomes.

Methods: In a nested case-control study, vaginal swabs from 79 pregnant women, i.e., 28 T. vaginalis-positive (cases) and 51 T. vaginalis-negative (controls), were screened by quantitative PCR for Adenovirus 1 and 2, Cytomegalovirus, Herpes Simplex Virus 1 and 2, Chlamydia trachomatis, Escherichia coli, Haemophilus ducreyi, Mycoplasma genitalium, M. hominis, candidatus M. girerdii, Neisseria gonorrhoeae, Streptococcus agalactiae, Treponema pallidum, Ureaplasma parvum, U. urealyticum, and Candida albicans. Additionally, we determined whether women with pathogens highly associated with $T$. vaginalis had distinct clinical signs and symptoms compared to women with T. vaginalis mono-infection.

Results: M. hominis was independently associated with T. vaginalis (adjusted odds ratio $=6.8,95 \%$ Cl: $2.3-19.8$ ). Moreover, M. genitalium and Ca M. girerdii were exclusively detected in women with $T$. vaginalis $(P=0.002$ and $P=0.001)$, respectively. Four of the six women co-infected with T. vaginalis and Ca M. girerdii complained of vaginal itching, compared to only 4 out of the 22 women infected with T. vaginalis without Ca M. girerdii $(P=0.020)$.

Conclusion: We confirm M. hominis as a correlate of T. vaginalis in our population, and the exclusive association of both M. genitalium and Ca. M. girerdii with T. vaginalis. Screening and treatment of these pathogens should be considered.
\end{abstract}

Keywords: Trichomonas, Mycoplasma hominis, M. Genitalium, M. Girerdii, Kenya, Pregnant, STIs

\section{Background}

Sexually transmitted infections (STIs) constitute a huge proportion of the most prevalent acute infections globally [1]. The most prevalent curable sexually transmitted pathogens include Chlamydia trachomatis, Neisseria gonorrhoeae, Treponema pallidum subspecies pallidum (syphilis) and Trichomonas vaginalis. These four pathogens are associated with acute conditions like genital/

\footnotetext{
* Correspondence: schengo@kemri-wellcome.org

${ }^{1}$ Kenya Medical Research Institute, Centre for Geographic Medicine Research

- Coast, Kenya Medical Research Institute, P.O. Box 230, Kilifi, Kenya

${ }^{2}$ Laboratory Bacteriology Research, Faculty of Medicine and Health Sciences,

Ghent University, De Pintelaan, 185 Ghent, Belgium

Full list of author information is available at the end of the article
}

anorectal/oral ulceration, cervicitis-endometritis, vaginal/urethral discharge, and urethritis. They can also cause critical complications and long term sequelae, which includes oophoritis, salpingitis, pelvic inflammatory disease, ectopic pregnancy, infertility, neurological disease, neonatal death, premature delivery and blindness [2]. Another public health concern is the association of STIs with the augmented possibility of HIV acquisition and transmission [3].

Of the four most prevalent curable STIs, $T$. vaginalis is globally the most prevalent pathogen [1], with a prevalence of up to $11.5 \%$ among women in sub-Sahara Africa [1]. Although there is a wealth of data regarding the

(c) The Author(s). 2018 Open Access This article is distributed under the terms of the Creative Commons Attribution 4.0 International License (http://creativecommons.org/licenses/by/4.0/), which permits unrestricted use, distribution, and reproduction in any medium, provided you give appropriate credit to the original author(s) and the source, provide a link to the Creative Commons license, and indicate if changes were made. The Creative Commons Public Domain Dedication waiver (http://creativecommons.org/publicdomain/zero/1.0/) applies to the data made available in this article, unless otherwise stated. 
clinical presentation and global burden of $T$. vaginalis [4], studies assessing associations of $T$. vaginalis with other genital pathogens are scarce, although it has been intimated that $T$. vaginalis has a unique symbiotic relationship with Mycoplasma hominis (M. hominis) [5]. T. vaginalis is also associated with an increase in vaginal $\mathrm{pH}[6]$ which may influence the composition of the associated vaginal microbial community and is strongly associated with bacterial vaginosis (BV) [6]. Less appreciated pathogens like $M$. hominis, $M$. genitalium, Ureaplasma parvum and $U$. urealyticum are increasingly being associated with adverse pregnancy outcomes as well as respiratory infections in neonates [7].

STI screening programs and STI research mostly focus on the four most common curable STIs, whereas other pathogens, associated with similar urogenital syndromes, remain undiagnosed and/or untreated. Detection of urogenital pathogens that may be associated with $T$. vaginalis may be important as it might have an effect on the clinical presentation and management and the long-term outcome of those infections. Here, we assess the occurrence of specific urogenital species in T. vaginalis-positive (cases) and T. vaginalis-negative (controls) among pregnant women in Kilifi, Kenya.

\section{Methods}

\section{Study setting}

Kenya, an East African country, is divided administratively into 47 counties. Kilifi County, which lies along the Indian Ocean Coast, is one of the poorest and is typical of a rural equatorial Africa setting. Among pregnant women in Kilifi the HIV prevalence is estimated to be $6.4 \%$, for chlamydia $14.9 \%$, for gonorrhea 1.0 and $7.4 \%$ for trichomoniasis [8].

From July till September 2015, we carried out a curable STI study at the prenatal care clinic of Kilifi County Hospital, Kenya. The key objective of the curable STI study was to illustrate the prevalence and predictors of curable sexually transmitted infections (STIs) among 350 pregnant women attending the prenatal care clinic [8]. The eligibility criteria for the curable STI study included: residing in the Kilifi Health and Demographic Surveillance area, age 18-45 years, willingness to undergo free STI and bacterial vaginosis (BV) screening procedures, gestation $\geq 14$ weeks, and willing to give written informed consent. This study presents a secondary aim of the curable STI study, which is to describe urogenital pathogen correlates of T. vaginalis among pregnant women in Kilifi, Kenya.

For the above-described curable STI study, a nurse at the prenatal care clinic collected vaginal secretions from the vaginal introitus using two sterile cotton swabs. The first vaginal swab was used for T. vaginalis detection using the InPouch system (BioMed Diagnostics, White City, Oregon, USA), a highly specific and sensitive device containing a fluid medium supporting the growth of $T$. vaginalis and allowing microscopic observation of $T$. vaginalis. The inoculated InPouch was transported to the laboratory within $15 \mathrm{~min}$ for direct microscopy, and incubation at $37{ }^{\circ} \mathrm{C} \pm 1{ }^{\circ} \mathrm{C}$. Daily microscopic observation (at both $\times 10$ and $\times 40$ magnification, for six fields) of the InPouch system was performed by qualified technicians. Motile trichomonads within 5 days of culture were indicative of being positive for $T$. vaginalis.

Same day treatment was offered for women who were determined to be positive for $T$. vaginalis by means of direct microscopy. For women whose culture turned positive but were negative for $T$. vaginalis on direct microscopy, they were contacted to return to the clinic for treatment the moment the culture turned positive. Secnidazole $2 \mathrm{~g}$ statim was administered as treatment, and participants were also asked to refer their sexual partner(s) to the clinic for treatment or were given the same medication to take to their sexual partner(s).

The second swab had its shaft broken by bending the shaft against the neck of a sterile, labeled $2 \mathrm{ml}$ Eppendorf tube, the tube containing the swab tip was closed and transferred to the laboratory where it was immediately stored at $-80^{\circ} \mathrm{C}$. No transport or freezing medium was added prior to storage.

Specimens for this case-control study are derived from the stored swabs from the curable STI study, published previously [7]. Because of financial and logistic constraints we could process only a subset of the 350 vaginal swabs from the main study. Vaginal swabs from 79 pregnant women were divided in two groups for analysis, i.e., those from women positive for T. vaginalis (cases) and those from women negative for T. vaginalis (controls) as determined by PCR. Controls were age-matched $(+/-5$ years) and all were bacterial vaginosis (BV) negative by Nugent score, largely matching the cases because only 4 out of the $28 \mathrm{TV}+$ cases were BV+. Selection of controls was guided by being TV negative. The swabs of 51 women selected as controls were not significantly different from the swabs of the other 271 women not selected as controls (Additional file 1: Table S1).

\section{DNA extraction}

Before DNA extraction, which was performed in Kilifi, the frozen swabs were thawed at room temperature (approximately $25{ }^{\circ} \mathrm{C}$ ) for $30 \mathrm{~min}$. Extraction was performed using the QIAamp DNA Mini Kit (Qiagen, Hilden, Germany) according to manufacturer's instructions and $160 \mu \mathrm{l}$ of eluted DNA was transferred to Eppendorf tubes and frozen at $-80{ }^{\circ} \mathrm{C}$ until shipment to the Laboratory of Bacteriology Research (LBR, Ghent University, Belgium). Shipment was performed using shipping boxes filled with dry ice $\left(-78.5^{\circ} \mathrm{C}\right)$. Once at the LBR the Eppendorf tubes with DNA-extracts were transferred 
back to $-80{ }^{\circ} \mathrm{C}$ until molecular analysis was performed. No thawing and freezing occurred after freezing the DNA extract until the point of molecular testing in the laboratory in Belgium.

\section{Quantitative PCR}

Most quantitative PCRs were performed using a highly sensitive and specific TaqMan ${ }^{\circ}$ Array Card (TAC), developed at AZ Sint-Jan Brugge-Oostende, Belgium. The array card was used for detecting Chlamydia trachomatis (including Lymphogranuloma venereum (LGV) serovars L1-L2-L3), Neisseria gonorrhoeae, Haemophilus ducreyi, Mycoplasma genitalium (including M. genitalium macrolide resistance-mediating mutations A2058G, A2059G, A2058T, A2058C in region V of the 23S rRNA gene), $M$. hominis, Ureaplasma parvum, $U$. urealyticum, Treponema pallidum, Herpes Simplex virus-1/- 2 (HSV 1/2), adenoviruses, Cytomegalovirus (CMV), and $T$. vaginalis. The assay has multiple genetic targets per pathogen in order to maximize both specificity and sensitivity [9]. Samples were determined to be positive for a particular species on the $\mathrm{TaqMan}^{\circ}$ Array Card (TAC), only in case the assay was positive for two independent PCR targets of that species. Sample quality was assessed by amplification of human DNA, to evaluate the quantity of epithelial cells recovered by the swab.

Further individual qPCRs were performed at the LBR for Candida albicans [10], Escherichia coli [11] and Streptococcus agalactiae $[11,12]$. The LightCycler 480 platform and the LightCycler 1480 Software Version 1.5 (Roche) were used for the amplification, detection and quantification. Each qPCR was performed in a final volume of $10 \mu \mathrm{l}$ of which $2 \mu \mathrm{l}$ of DNA extract or $2 \mu \mathrm{l}$ of a negative control (HPLC water) or $2 \mu \mathrm{l}$ of a positive control. All the specific primers and probes were synthesized by Eurogentec, Liège, Belgium. Specific qPCR details are provided in Additional file 1: Table S2.

The procedures as described by Cools et al. [10] were adopted for the construction of qPCR standard curves. Briefly, DNA was extracted from overnight cultures of $C$. albicans ATCC 90028 grown on Sabouraud agar (Becton Dickinson, Erembodegem, Belgium) and of E. coli ATCC 25922 or S. agalactiae LMG $14694^{\mathrm{T}}$ grown on TSA $+5 \%$ sheep blood (Becton Dickinson). All colonies were collected from the plate and re-suspended in $1 \mathrm{ml}$ of saline. The manufacturer's instructions of the High Pure PCR Template Preparation Kit (Roche Applied Science, Basel, Switzerland) were followed to extract DNA from this suspension. DNA-concentration was determined by means of Nanodrop and the number of genomes was calculated. A tenfold dilution series in HPLC-grade water was made to establish for each dilution the number of Cqs needed to pass the detection threshold. Using these data, a regression curve was constructed.

\section{PCR for T. vaginalis and for Candidatus Mycoplasma} girerdii (Ca. M. girerdii)

PCRs for $T$. vaginalis targeting the actin gene, using outer primers, previously used in a nested PCR [13] and yielding a fragment of $1100 \mathrm{bp}$ and for Ca. M. girerdii, yielding a fragment of 594 bp [14], were carried out on the ABI Veriti thermocycler platform (ThermoFisher Scientific, Waltham, Massachusetts). The primers were synthesized by Eurogentec, Liège, Belgium. Amplified fragments were visualized under UV light after agarose gel electrophoresis and $\mathrm{EtBr}$ staining.

Details of these species-specific PCRs are summarized in Additional file 1: Table S2.

\section{Sequencing}

Sequencing of PCR amplicons was carried out by GATC Biotech (Constance, Germany) to confirm specificity of the PCR products. Sequencing was done using the forward PCR primers (Additional file 1: Table S2). Sequences were cleaned using Chromas Lite version 2.1 (Technelysium, Brisbane, Australia). BLAST was performed on the sequences to confirm the identity.

\section{Data analysis}

Epidemiological data were analyzed using StataCorp. 2013. Stata Statistical Software: Release 13 (College Station, TX: StataCorp LP). Prevalence of urogenital pathogens were computed with $95 \%$ confidence intervals (CIs). Associations between T. vaginalis positivity and socio-demographic, hygienic and behavioral characteristics were calculated using the $\mathrm{x} 2$ test. To build a multivariate model of urogenital species associated with $T$. vaginalis, we first carried out univariate regression analysis. For computation of odds ratios (ORs), we replaced all zero values in cells by the value ' 0.5 ', as suggested by Deeks \& Higgins [13].

Species that were significantly associated with $T$. vaginalis in univariate regression analysis $P$-value $\leq 0.1$ were selected for multivariate logistic regression analysis. Associations in the final multivariate model were expressed as adjusted odds ratios (AORs) with $p$-values $\leq 0.05$ considered significant. We further assessed whether pathogens, significantly associated with $T$. vaginalis infection, had an implication on the clinical presentation.

\section{Results}

A total of 23 out of 350 samples (6.5\%) were positive by InPouch culture for Trichomonas vaginalis, of which eight (34.8\%) were initially positive on direct microscopy. The T. vaginalis-specific PCR [13] detected one additional case of $T$. vaginalis from a sample that was negative by InPouch culture but positive by the TAC assay, which found four more positive samples. In summary, sensitivity of direct microscopy, of $T$. vaginalis 
InPouch culture and of T. vaginalis-specific PCR were respectively $28.6,82.1$ and $85.7 \%$, when compared to the TAC assay.

Distribution of age, religion, education level, marital status, parity, gestational age and number of lifetime sex partners were similar among cases and controls (Table 1). HIV and BV status was different between the two groups by our case-control study design (Table 2), but the resulting overall difference was minimal, i.e. $0 \mathrm{HIV}$ and $0 \mathrm{BV}$ cases in the control group compared to 3 HIV-positives (10.7\%) and 4 BV-positives (14.3\%) in the Trichomonas positive group. Moreover, we could show that these differences had no influence on the species associated with $T$. vaginalis (Additional file 1: Tables S3 and S4). Additionally, occupation was also significantly different (Table 1).

\section{Prevalence of urogenital species}

The prevalence of the co-infecting urogenital species is indicated in Table 2. Adenovirus, Haemophilus ducreyi, Neisseria gonorrhoeae and Treponema pallidum were

Table 1 Characteristics of Trichomonas vaginalis qPCR positive women (cases) and T. vaginalis qPCR negative women (controls)

\begin{tabular}{|c|c|c|c|}
\hline Characteristic & $\begin{array}{l}\text { Cases (\%) } \\
N=28\end{array}$ & $\begin{array}{l}\text { Controls (\%) } \\
N=51\end{array}$ & $\begin{array}{l}x 2 \times \\
P \text {-value }\end{array}$ \\
\hline \multicolumn{4}{|l|}{ Age group (Years) } \\
\hline $18-24$ & 42.9 & 31.4 & \multirow[t]{2}{*}{0.307} \\
\hline$\geq 25$ & 57.1 & 68.6 & \\
\hline \multicolumn{4}{|l|}{ Religion } \\
\hline Christian & 64.3 & 68.6 & \multirow[t]{3}{*}{0.383} \\
\hline Muslim & 10.7 & 17.7 & \\
\hline Other/None & 25.0 & 13.7 & \\
\hline \multicolumn{4}{|l|}{ Education } \\
\hline None & 21.4 & 23.5 & \multirow[t]{3}{*}{0.946} \\
\hline Primary & 60.7 & 56.9 & \\
\hline Secondary/Tertiary & 17.9 & 19.6 & \\
\hline \multicolumn{4}{|l|}{ Employment status } \\
\hline Employed/self-employed & 50.0 & 72.6 & \multirow[t]{2}{*}{0.045} \\
\hline Unemployed & 50.0 & 27.5 & \\
\hline \multicolumn{4}{|l|}{ Parity } \\
\hline 0 & 25.0 & 35.3 & \multirow[t]{3}{*}{0.234} \\
\hline $1-2$ & 46.4 & 25.4 & \\
\hline $3+$ & 28.6 & 37.3 & \\
\hline \multicolumn{4}{|l|}{ Gestational age (weeks) } \\
\hline $14-27$ & 57.1 & 58.8 & \multirow[t]{2}{*}{0.885} \\
\hline$\geq 28$ & 42.9 & 41.2 & \\
\hline \multicolumn{4}{|c|}{ Number of lifetime sex partners } \\
\hline 1 & 82.1 & 90.2 & \multirow[t]{2}{*}{0.303} \\
\hline $3+$ & 21.7 & 9.8 & \\
\hline
\end{tabular}

In bold: significantly associated, i.e., $P \leq 0.05$ not detected and are not reported in Table 1. Ureaplasma parvum was the most prevalent at $74.7 \%$ ( $95 \%$ Confidence interval (CI): 63.6-83.8), followed by $U$. urealyticum at $48.1 \%$ (CI: 36.7-59.6).

\section{Univariate and multivariate association analysis}

Although, M. genitalium and Ca. M. girerdii, had generally a low prevalences of respectively 6.3 and $7.6 \%$, the two were exclusively detected in women with $T$. vaginalis (Chi-square test: $\chi^{2}=9.7, \mathrm{df}=1, P<0.002$ and $\chi^{2}=11.8$, $\mathrm{df}=1, P<0.001$, respectively). Both $M$. genitalium and Ca. M. girerdii were significantly associated with $T$. vaginalis on univariate analysis but not on multivariable analysis (Table 2). None of the samples for which $M$. genitalium could be detected showed macrolide resistance-associated mutations. In a univariate regression analysis, $M$. hominis and $U$. urealyticum were significantly associated with $T$. vaginalis (crude odds ratio $(C O R)=7.3$; 95\% CI: 2.6-20.5 and COR $=2.2$; 95\% CI: 0.9-5.7, respectively). We detected $M$. hominis from the vaginal DNA extracts of approximately $70 \%$ of women with $T$. vaginalis. $M$. hominis was also independently associated with $T$. vaginalis in a multivariate regression analysis (adjusted odds ratio $(\mathrm{AOR})=6.8 ; 95 \%$ CI: $2.3-19.8$ )

Tables 3, 4 and 5 compare clinical signs and symptoms among $T$. vaginalis-infected women co-infected or not with M. hominis (Table 3), or with Ca M. girerdii (Table 4) or with M. genitalium (Table 5).

Women co-infected with $T$. vaginalis and Ca. M. girerdii were more likely to report vaginal itching compared to $T$. vaginalis-positive women not co-infected with Ca. M. girerdii $(66.8 \%$ vs. $18.2 \%(p=0.020))$. There was no difference in clinical presentation of T. vaginalis-infected pregnant women co-infected with $M$. hominis or with $M$. genitalium, compared to those not co-infected with these species.

Of the five participants that had M. genitalium and the six that had Ca M. girerdii, only one participant had a co-infection with $M$. genitalium and $\mathrm{Ca} M$. girerdii. However, $M$. hominis was always present in vaginal samples from which $M$. genitalium and $\mathrm{Ca} M$. girerdii were detected. Due to the detection of CMV, HSV 1/ 2, $M$. genitalium and Ca. M. girerdii exclusively in cases or controls, the species were excluded from the regression model.

\section{Discussion}

Our results indicate that women with Trichomonas vaginalis $(n=28)$ have a high rate $(71.4 \%)$ of co-infection with Mycoplasma hominis compared to only $25.5 \%$ of 51 women not infected with $T$. vaginalis. Comparable rates of co-infection have been reported by Becker et al. [15], i.e., $56.7 \%$ in Brazil and by Xiao et al. [16], i.e., $50.0 \%$ in China. Rappelli et al. [17] reported much higher rates 
Table 2 Prevalence and co-occurrence of urogenital species among 28 Trichomonas vaginalis-positive and 51 T. vaginalis-negative women

\begin{tabular}{|c|c|c|c|c|c|c|}
\hline \multirow[t]{2}{*}{ Species } & \multirow{2}{*}{$\begin{array}{l}\text { Overall prevalence } \\
(N=79) \\
(95 \% \mathrm{Cl})\end{array}$} & \multirow{2}{*}{$\begin{array}{l}\% \text { TV } \\
+/ \% \text { TV- }\end{array}$} & \multicolumn{2}{|l|}{ Univariate analysis } & \multicolumn{2}{|c|}{ Multivariate analysis } \\
\hline & & & $\begin{array}{l}\text { COR } \\
(95 \% \mathrm{Cl})\end{array}$ & $P$-value & $\begin{array}{l}\text { AOR } \\
(95 \% \mathrm{Cl})\end{array}$ & $P$-value \\
\hline Candida albicans & $24.1(15.1-35.0)$ & $32.1 / 19.6$ & $1.9(0.7-5.6)$ & 0.216 & - & - \\
\hline Chlamydia trachomatis & $13.9(7.2-23.5)$ & $21.4 / 9.8$ & $2.5(0.7-9.1)$ & 0.163 & - & - \\
\hline Escherichia coli & $27.8(18.3-39.1)$ & $35.7 / 23.5$ & $1.8(0.7-4.9)$ & 0.251 & - & - \\
\hline Mycoplasma genitalium & $6.3(2.1-14.2)$ & 17.9/0.0 & $5.0(0.3-94.3)^{*}$ & 0.002 & & \\
\hline Ca. Mycoplasma girerdii & $7.6(2.8-15.8)$ & $21.4 / 0.0$ & $4.3(0.2-78.4)^{*}$ & 0.001 & & \\
\hline Mycoplasma hominis & $41.8(30.8-53.4)$ & $71.4 / 25.5$ & $7.3(2.6-20.5)$ & $<0.001$ & $6.8(2.3-19.8)$ & $<0.001$ \\
\hline Streptococcus agalactiae & $11.4(5.3-20.5)$ & 7.1/13.7 & $0.5(0.1-2.5)$ & 0.386 & - & - \\
\hline Ureaplasma parvum & 74.7 (63.6-83.8) & $78.9 / 72.6$ & $1.4(0.5-4.1)$ & 0.557 & - & - \\
\hline Ureaplasma urealyticum & $48.1(36.7-59.6)$ & $60.7 / 41.2$ & $2.2(0.9-5.7)$ & 0.099 & $1.3(0.4-3.8)$ & 0.624 \\
\hline Cytomegalovirus & $1.3(0-6.9)$ & $0.0 / 2.0$ & $1.7(6.6-4210.4)^{*}$ & 0.456 & & \\
\hline HIV & $3.8(0.7-10.7)$ & $10.7 / 0.0$ & $7.9(0.4-158.5)^{*}$ & 0.017 & & \\
\hline HSV 1, HSV 2 & $2.5(0.3-8.8)$ & $7.1 / 0.0$ & $11.1(0.5-238.6)^{*}$ & 0.053 & & \\
\hline Bacterial vaginosis & $5.1(1.4-12.5)$ & $14.3 / 0.0$ & $6.2(0.3-118.4)^{*}$ & 0.006 & & \\
\hline
\end{tabular}

TV Trichomonas vaginalis, HIV human immunodeficiency virus, HSV1 herpes simplex virus type 1; herpes simplex virus type 2, COR crude odds ratio, AOR adjusted odds ratio

in bold: significantly associated, i.e., $P \leq 0.05^{*}$ Separate computation not included in multivariable model

(94.3\%) of co-infection among Italian, Mozambican, and Angolan women. M. hominis has been shown to have a symbiotic relationship with $T$. vaginalis. Owing to its small genome, this bacterial species is strongly dependent on host cell metabolism. M. hominis has the ability to enter trichomonad cells by endocytosis and to multiply in coordination with the protozoan [5]. We could not establish differences in clinical presentation of women co-infected with both $T$. vaginalis and $M$. hominis as compared to those infected with only $T$. vaginalis, in agreement with previous data [18]. As such, at present, co-infection of $T$. vaginalis with $M$. hominis seems to be of limited clinical relevance, also because antibiotic treatment of the former

Table 3 Clinical signs/symptoms among women co-infected with Trichomonas vaginalis and Mycoplasma hominis versus T. vaginalis only

\begin{tabular}{|c|c|c|c|}
\hline Clinical sign or symptom & $\begin{array}{l}\text { TV with MH } \\
N=20(\%)\end{array}$ & $\begin{array}{l}\text { TV without MH } \\
N=8(\%)\end{array}$ & $\begin{array}{l}x 2 \\
P \text {-value }\end{array}$ \\
\hline Dyspareunia & 40.0 & 37.5 & 0.903 \\
\hline Dysuria & 30.0 & 37.5 & 0.701 \\
\hline Foul smelling vaginal odor & 30.0 & 12.5 & 0.334 \\
\hline Genital ulcers & 15.0 & 12.5 & 0.864 \\
\hline Genital warts & 10.0 & 0.0 & 0.353 \\
\hline Lower abdominal pain & 40.0 & 25.0 & 0.454 \\
\hline Vaginal discharge & 75.0 & 62.5 & 0.508 \\
\hline Vaginal itching & 30.0 & 25.0 & 0.791 \\
\hline
\end{tabular}

MH Mycoplasma hominis, TV Trichomonas vaginalis will probably consecutively diminish the presence of the latter.

The pathogenic potential of $M$. genitalium among pregnant women in Kenya has not been extensively investigated probably because its prevalence is overshadowed by a higher prevalence of other STIs, as was the case in this study. Our results indicate that in our population M. genitalium was strongly associated with $T$. vaginalis $(p=0.002)$. Given the presence of $M$. genitalium exclusively in women with $T$. vaginalis infection, screening and treatment of women for $T$. vaginalis might also at once reduce the prevalence of $M$. genitalium. Although macrolide resistance associated mutations among $M$. genitalium strains are on

Table 4 Clinical signs/symptoms among women co-infected with Trichomonas vaginalis and Ca Mycoplasma girerdii versus T. vaginalis only

\begin{tabular}{llll}
\hline Clinical sign or symptom & $\begin{array}{l}\text { TV with Ca MG } \\
N=6(\%)\end{array}$ & $\begin{array}{l}\text { TV without Ca MG } \\
N=22(\%)\end{array}$ & $\begin{array}{l}\text { X2 } \\
P \text {-value }\end{array}$ \\
\hline Dyspareunia & 50.0 & 36.4 & 0.544 \\
Dysuria & 50.0 & 27.3 & 0.291 \\
Foul smelling vaginal odor & 33.3 & 22.7 & 0.595 \\
Genital ulcers & 16.7 & 13.6 & 0.851 \\
Genital warts & 0.0 & 9.1 & 0.443 \\
Lower abdominal pain & 50.0 & 32.8 & 0.410 \\
Vaginal discharge & 100.0 & 63.6 & 0.081 \\
Vaginal itching & 66.7 & 18.2 & $\mathbf{0 . 0 2 0}$ \\
\hline
\end{tabular}

Ca MG Candidatus Mycoplasma girerdii, TV Trichomonas vaginalis; in bold: significantly associated 
Table 5 Clinical signs/symptoms among women co-infected with Trichomonas vaginalis and Mycoplasma genitalium versus T. vaginalis only

\begin{tabular}{llll}
\hline Clinical sign or symptom & TV with MG & $\begin{array}{l}\text { TV without MG } \\
N=5(\%)\end{array}$ & (X2).P-value \\
\hline Dyspareunia & 20.0 & 43.5 & \\
Dysuria & 20.0 & 34.8 & 0.330 \\
Foul smelling vaginal odor & 40.0 & 21.7 & 0.521 \\
Genital ulcers & 0.0 & 17.4 & 0.393 \\
Genital warts & 0.0 & 8.7 & 0.314 \\
Lower abdominal pain & 40.0 & 34.8 & 0.494 \\
Vaginal discharge & 80.0 & 69.6 & 0.825 \\
Vaginal itching & 0.0 & 34.8 & 0.640 \\
\hline
\end{tabular}

MG Mycoplasma genitalium, TV Trichomonas vaginalis

the rise, as was recently shown among female sex workers in Belgium [19], macrolide resistance-associated mutations could not be detected in any of the five samples positive for M. genitalium and therefore our results, although based on a very small sample, suggest that macrolides can still be used for treatment of $M$. genitalium in this population in Kilifi, Kenya.

To our knowledge, this is the first report of Ca. M. girerdii in Africa. In agreement with the two initial reports on the prevalence of Ca. M. girerdii [20,21], we found it to be strongly associated with $T$. vaginalis $(p=0.001)$. Fettweis et al. [20], using a pyrosequencing approach, were the first to detect Ca. M. girerdii DNA in the vaginal swabs of a few women not infected with $T$. vaginalis, as assessed with qPCR, although in our study, Ca. $M$. girerdii was found only in $T$. vaginalis positive women. A recent report by Costello et al. [22] is in support of the close association of Ca. M. girerdii and T. vaginalis as they recovered $\mathrm{Ca}$. M. girerdii and $T$. vaginalis genomes from the saliva of a premature infant.

Our data suggest that $T$. vaginalis-positive women, co-infected with Ca. M. girerdii, were more likely to report vaginal itching compared to $T$. vaginalis mono-infected women. Future studies should elucidate the nature of the interaction of these two pathogens and the effect that co-infection may have on clinical presentation.

$U$. parvum and $U$. urealyticum are commonly isolated from the vaginal microbiome of asymptomatic pregnant women [23], as was the case in our study. Although detection of $U$. parvum has been associated with preterm birth [24], opinions differ with regard to the need to screen and treat Ureaplasma spp. infection during pregnancy, since its presence often represents colonization rather than infection [25]. Our data did not show any association between $T$. vaginalis and either $U$. parvum or $U$. urealyticum.

C. trachomatis was highly prevalent $(13.9 \%)$ in our study. All isolates were non-LGV strains, but were not associated with $T$. vaginalis infection. Our results on urogenital carriage of Candida, E. coli, and GBS indicate that the three were not associated with $T$. vaginalis infection, either. While the prevalence of Candida in our study was higher than that reported in a cross-sectional study by Cools et al. [11] among women in Kenya, Rwanda and South-Africa, our prevalence for E. coli and GBS is comparable to what they reported.

Our study had some limitations. First, it only included a relatively small sample size of pregnant women limiting the precision of our prevalence estimates. Furthermore, only BV negative samples were included in the control arm, which may represent a bias on the interpretation of the results. Among the 28 T. vaginalis-positive women, only four were positive for BV and excluding them in the analysis does not affect the results (Additional file 1: Table S3). It should be noted that our T. vaginalis/BV co-infection rate of $14 \%$ was comparable to that observed in a recent study, i.e. $17.5 \%$ among HIV + women [26]. Finally, no internal control was added during the DNA extraction process, so inefficient genome extraction or (partial) PCR inhibition could not be documented. However, sample adequacy was evaluated by detecting a minimal level of human DNA present in the sample, which was the case for all samples, moreover none of the samples that were culture-positive for $T$. vaginalis were missed by PCR.

\section{Conclusion}

We observed notable prevalence of urogenital micro-organisms, pathogens and colonizing germs among pregnant women which emphasizes the need for laboratory testing and treatment to avoid unfavorable pregnancy outcomes. We confirm $M$. hominis as a correlate of $T$. vaginalis in our population, but the most salient finding was the exclusive association of both $M$. genitalium and Ca. M. girerdii with $T$. vaginalis. The latter finding ought to be further addressed using a larger sample size.

\section{Additional file}

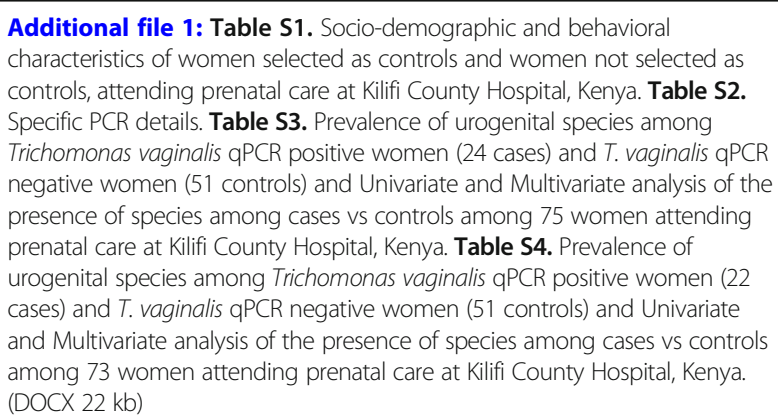

Abbreviations

BV: Bacterial vaginosis; Ca. M. girerdii: Candidatus Mycoplasma girerdii; M. genitalium: Mycoplasma genitalium; M. hominis: Mycoplasma hominis; 
PCR: Polymerase chain reaction; STls: Sexually transmitted infections; $T$. vaginalis: Trichomonas vaginalis; TAC: TaqMan ${ }^{\circledR}$ Array Card; U. parvum: Ureaplasma parvum; U. urealyticum: Ureaplsama urealyticum

\section{Acknowledgements}

We would like to thank the study participants. A special thanks to all nurses at the prenatal care clinic of the Kilifi County Hospital. We also wish to acknowledge Sarah Kioko who was a field worker in the curable STI study. This manuscript was submitted for publication with permission from the Director of the Kenya Medical Research Institute (KEMRI).

\section{Funding}

This research has been supported by a PhD Scholarship to Simon C. Masha from the Belgian Development Cooperation through VLIR-UOS. The funders had no role in the design of the study and collection, analysis, and interpretation of data and in writing the manuscript. The views expressed here are those of the authors and do not necessarily represent the views of the Belgian Development Cooperation

\section{Availability of data and materials}

All data generated or analysed during this study are included in this published article (and its Additional files).

\section{Authors' contributions}

SCM, PC, EJS and MV designed the study. SCM supervised the field data collections. SCM, PC, PD and MR conducted laboratory analysis. SCM performed data management, and analysis. All authors contributed to the interpretation of results. SCM wrote the initial manuscript draft, PC, PD, MR, EJS and MV critically reviewed the manuscript. MV gave overall supervision, and including on the manuscript preparation. All authors read and approved the final version of the manuscript and agree to be accountable for all aspects of the work.

\section{Ethics approval and consent to participate}

We obtained ethical approval from the Kenya Medical Research Institute Scientific and Ethics Review Unit (\#3022). Written informed consent was obtained from all the participants.

\section{Consent for publication}

Not applicable

\section{Competing interests}

The authors declare that they have no competing interests.

\section{Publisher's Note}

Springer Nature remains neutral with regard to jurisdictional claims in published maps and institutional affiliations.

\footnotetext{
Author details

'Kenya Medical Research Institute, Centre for Geographic Medicine Research - Coast, Kenya Medical Research Institute, P.O. Box 230, Kilifi, Kenya. ${ }^{2}$ Laboratory Bacteriology Research, Faculty of Medicine and Health Sciences, Ghent University, De Pintelaan, 185 Ghent, Belgium. ${ }^{3}$ Faculty of Pure and Applied Sciences, Department of Biological Sciences, Pwani University, Kilifi, Kenya. ${ }^{4}$ Department of Laboratory Medicine, Medical Microbiology, AZ St-Jan Brugge-Oostende, Bruges, Belgium.
}

Received: 29 January 2018 Accepted: 23 October 2018 Published online: 06 November 2018

\section{References}

1. Newman L, Rowley J, Vander Hoorn S, et al. Global estimates of the prevalence and incidence of four curable sexually transmitted infections in 2012 based on systematic review and global reporting. PLoS One. 2015;10: e0143304.

2. Vos T, Barber RM, Bell B, et al. Global, regional, and national incidence, prevalence, and years lived with disability for 301 acute and chronic diseases and injuries in 188 countries, 1990 -2013: a systematic analysis for the global burden of disease study 2013. Lancet. 2015;386:743-800.

3. McClelland RS, Sangare L, Hassan WM, et al. Infection with Trichomonas vaginalis increases the risk of HIV-1 acquisition. J Infect Dis. 2007;195: 698-702.
4. Petrin D, Delgaty $K$, Bhatt $R$, et al. Clinical and microbiological aspects of Trichomonas vaginalis. Clin Microbiol Rev. 1998;11:300-17.

5. Fichorova R, Fraga J, Rappelli $P$, et al. Trichomonas vaginalis infection in symbiosis with Trichomonasvirus and Mycoplasma. Res Microbiol. 2017; 168:882-91.

6. Azargoon A, Darvishzadeh S. Association of bacterial vaginosis, Trichomonas vaginalis, and vaginal acidity with outcome of pregnancy. Arch Iranian Med. 2006;9:213-7.

7. Donders GGG, Ruban K, Bellen G, et al. Mycoplasma/Ureaplasma infection in pregnancy: to screen or not to screen. J Perinat Med. 2017;45(5):505-15.

8. Masha SC, Wahome E, Vaneechoutte M, et al. High prevalence of curable sexually transmitted infections among pregnant women in a rural county hospital in Kilifi, Kenya. PloS One. 2017;12:e0175166.

9. Steensels D, Reynders M, Descheemaeker $P$, et al. Clinical evaluation of a multi-parameter customized respiratory TaqMan ${ }^{\oplus}$ array card compared to conventional methods in immunocompromised patients. J Clin Vir. 2015;72:36-41.

10. Duyvejonck H, Cools P, Decruyenaere J, et al. Validation of High Resolution Melting Analysis (HRM) of the amplified ITS2 region for the detection and identification of yeasts from clinical samples: Comparison with culture and MALDI-TOF based identification. PLOS ONE. 2015;10:e0132149 Erratum in: PLoS One 2015; 10:e0139501.

11. Cools $P$, Jespers $V$, Hardy $L$, et al. A multi-country cross-sectional study of vaginal carriage of group B streptococci (GBS) and Escherichia coli in resource-poor settings: prevalences and risk factors. PLoS One. 2016;11: e0148052.

12. El Aila NA, Cools $P$, Deschaght $P$, et al. Strong correspondence of the vaginal and rectal load of group B streptococci in pregnant women. J Clin Gynecol Obst. 2013;2:61-7.

13. Deeks JJ, Higgins JPT. Statistical algorithms in review. Manager. 2010;5 http://training.cochrane.org/handbook/statistical-methods-revman5. Accessed 6 Jun 2018.

14. Crucitti T, Abdellati S, Van Dyck E, Buve A. Molecular typing of the actin gene of Trichomonas vaginalis isolates by PCR-restriction fragment length polymorphism. Clin Microbiol Infect. 2008;14:844-52.

15. Becker DD, dos Santos O, Frasson AP, et al. High rates of double-stranded RNA viruses and Mycoplasma hominis in Trichomonas vaginalis clinical isolates in South Brazil. Infect Genet Evol. 2015;34:181-7.

16. Xiao JC, Xie LF, Fang SL, et al. Symbiosis of Mycoplasma hominis in Trichomonas vaginalis may link metronidazole resistance in vitro. Parasit Res. 2006;100:123-30.

17. Rappelli P, Addis MF, Carta F, et al. Mycoplasma hominis parasitism of Trichomonas vaginalis. Lancet. 1998;352:1286.

18. Van Belkum A, Van der Meijden WI, Verbrugh HA, et al. A clinical study on the association of Trichomonas vaginalis and Mycoplasma hominis infections in women attending a sexually transmitted disease (STD) outpatient clinic. Immun Med Microbiol. 2001;32:27-32.

19. Coorevits $L$, Traen A, Binge $L$, et al. Macrolide resistance in mycoplasma genitalium from female sex workers in Belgium. J Glob Antimicrob Resist. 2018;12:149-52.

20. Fettweis JM, Serrano MG, Huang B, et al. An emerging Mycoplasma associated with trichomoniasis, vaginal infection and disease. PloS One. 2014;9:e110943.

21. Martin DH, Zozaya M, Lillis RA, et al. Unique vaginal microbiota that includes an unknown Mycoplasma-like organism is associated with Trichomonas vaginalis infection. J Infect Dis. 2013;207:1922-31.

22. Costello EK, Sun CL, Carlisle EM, et al. Candidatus Mycoplasma girerdii replicates, diversifies, and co-occurs with Trichomonas vaginalis in the oral cavity of a premature infant. Sci Rep. 2017;7:3764.

23. Taylor-Robinson D. Mollicutes in vaginal microbiology: Mycoplasma hominis, Ureaplasma urealyticum, Ureaplasma parvum and Mycoplasma genitalium. Res Microbiol. 2017;168:875-81.

24. Payne MS, Ireland DJ, Watts $\mathrm{R}$, et al. Ureaplasma parvum genotype, combined vaginal colonisation with Candida albicans, and spontaneous preterm birth in an Australian cohort of pregnant women. BMC Pregnancy Childbirth. 2016;16:312.

25. Donders GG, Ruban K, Bellen G, et al. Mycoplasma/Ureaplasma infection in pregnancy: to screen or not to screen. J Perinatal Med. 2017;45:505-15.

26. Gatski M, Martin DH, Clark RA, et al. Co-occurrence of Trichomonas vaginalis and bacterial vaginosis among HIV-positive women. Sex Transm Dis. 2011;38:163-6. 